Nouvelles perspectives en sciences sociales

Revue internationale de systémique complexe et d'études relationnelles

\title{
Instances audiovisuelles françaises et programmation : la place des études sur la représentation des minorités ethniques à la télévision
}

\section{Catherine Ghosn}

Volume 8, numéro 1, novembre 2012

URI : https://id.erudit.org/iderudit/1013923ar

DOI : https://doi.org/10.7202/1013923ar

Aller au sommaire du numéro

Éditeur(s)

Prise de parole

ISSN

1712-8307 (imprimé)

1918-7475 (numérique)

Découvrir la revue

Citer cet article

Ghosn, C. (2012). Instances audiovisuelles françaises et programmation : la place des études sur la représentation des minorités ethniques à la télévision. Nouvelles perspectives en sciences sociales, 8(1), 221-236.

https://doi.org/10.7202/1013923ar
Résumé de l'article

Associer " représentation de la diversité ethnique » et « télévision » dans une juste mesure ne relève-t-il pas d'une impossibilité? Pour répondre à cette question, nous considérons d'abord les fondements scientifiques, souvent venus de l'étranger, qui ont déterminé la prise en compte d'objets « hors normes ", différents de ceux habituellement traités en sciences sociales. En France, ces recherches restent majoritairement limitées au domaine universitaire et scientifique. Il faut attendre l'année 2000 puis l'année 2008 pour consulter les deux études menées par des chercheurs et destinées à des instances audiovisuelles. Quelles répercussions ces démarches et résultats scientifiques ont-ils concrètement sur la politique télévisuelle? Nous nous appuyons sur deux rapports rédigés par les instances audiovisuelles françaises pour évaluer dans quelles mesures ces intentions modifient effectivement le paysage audiovisuel. 


\title{
Instances audiovisuelles françaises et programmation : la place des études sur la représentation des minorités ethniques à la télévision
}

\author{
Catherine Ghosn \\ Université Paul Sabatier, Toulouse \\ Laboratoire d'Études et de recherches Appliquées \\ en Sciences Sociales (LERASS)
}

Cet article s'inscrit dans le cadre d'un travail de recherche mené sur la représentation des minorités ethniques à la télévision en Sciences de l'information et de la communication. Nous nous sommes principalement aidée de recherches en sociologie pour questionner les concepts de représentation (Isabelle Rigoni, Claire Cossée), de minorité ethnique (Marie-France Malonga, Eric Macé) et pour interroger la croyance généralement partagée que la télévision française reflète la société dans son ensemble, en prenant en compte ses diverses composantes. Dans ce cadre, la problématique consacrée au numéro de Nouvelles perspectives en sciences sociales, centrée sur l'homogénéisation et la différenciation, recoupe notre objet d'étude dans la mesure où elle interroge la possibilité de faire coexister deux états, deux concepts différents.

L'objet de notre étude consiste tout d'abord à analyser la production scientifique traitant de la représentation des minorités ethniques à la télévision en France, pour mesurer dans un 
second temps la manière dont les principales instances audiovisuelles françaises ont pris en compte cette production pour améliorer cette représentation sur le petit écran. Nous proposons de répondre à cette question en considérant d'abord les fondements scientifiques, souvent venus de l'étranger, qui ont déterminé la prise en compte d'objets " hors normes ", différents de ceux habituellement traités en sciences sociales. Pour quelle raison la France est-elle restée en retrait sur la thématique des minorités ethniques? Que propose-t-elle en termes de recherche et de publications en sciences sociales? Nous confrontons ensuite ces fondements épistémologiques à deux rapports émanant du Conseil Supérieur de l'Audiovisuel (CSA) et de France Télévisions, datés de 2010, pour évaluer dans quelles mesures les recherches en sciences sociales et la réflexion menée au sein des instances audiovisuelles peuvent se croiser, au même titre que les travaux menés aux États-Unis ou en Grande-Bretagne.

\section{Fondements scientifiques}

Les cultural studies et l'École de Francfort ont ceci en commun qu'elles ont aidé à porter un regard sur la notion de "différence " dans l'espace public - notion que nous rattachons dans cette étude à celle de minorité ethnique - en mettant en place une approche critique qui a entraîné une reconnaissance et une légitimité scientifique à ce concept. Parmi les publications traitant des cultural studies selon une approche communicationnelle, quelques points méritent d'être relevés pour analyser plus finement comment cette notion a été traitée dans l'espace médiatique $^{1}$.

Les cultural studies portent une dimension critique sur le lien généralement établi entre les cultures et le pouvoir. Apparues en Grande-Bretagne dans les années 1960, elles sont activement menées par, entre autres, Richard Hoggart (il fonde le Centre for

$1 \quad$ Armand Mattelart et Erik Neveu, Introduction aux cultural studies, Paris, La Découverte, 2003; Hervé Glevarec, Éric Macéet Éric Maigret, Cultural studies : anthologie, Paris, Armand Colin, 2008; Françoise Albertini et Nicolas Pélissier, Les Sciences de l'information et de la communication à la rencontre des cultural studies, Paris, L'Harmattan, 2009. 
Contemporary Cultural Studies (CCCS) à Birmingham en 1964), Stuart Hall (il succède à Richard Hoggart au CCCS), Charlotte Brunsdon (qui travaille sur le féminisme dans les médias). Ils ont permis de procéder à une approche transversale des cultures populaires et minoritaires au cours des années 1960 pour travailler sur leurs rapports avec la culture dominante, ont apporté des « objets pensables par l'Academia ${ }^{2}$ » et mis notamment en avant le concept de genres et d'identités ethniques. Mener une analyse sur les cultures populaires, les identités ouvrières, les appartenances ethniques et la problématique du racisme a permis d'élargir le champ d'étude et d'intégrer à la réflexion les éléments qui touchent au genre (Women's Studies Group, 1978). L'intérêt d'une telle approche a, par exemple, consisté à étudier les différences de consommation des programmes télévisuels entre les hommes et les femmes, ou encore à constater que la prédominance des personnages, en termes d'études ou de présence est majoritairement masculine. Les membres l'École de Francfort, en majorité des intellectuels allemands exilés aux États-Unis après l'arrivée au pouvoir du parti nazi (Theodor W. Adorno, Max Horkheimer, Walter Benjamin, Herbert Marcuse) élaborent une théorie critique (Kulturindustrie) sur l'industrialisation de la culture populaire, et partagent avec les cultural studies une disposition compréhensive dans l'étude des objets ainsi qu'une position critique sur le conformisme ou le légitimisme culturel, scientifique ${ }^{3}$.

Ces deux écoles, venant de l'étranger, reçoivent un accueil très réservé en France, surtout en ce qui concerne les cultural studies. Des raisons conjoncturelles principalement liées au contexte politique et social expliquent en partie les différences d'intérêt accordé à la problématique des minorités ethniques entre la

2 Erik Neveu, "Les Voyages des cultural studies ", L'Homme, vol. 187-188, $\mathrm{n}^{\text {os }}$ 3-4, 2008, p. 315.

3 Benjamin Walter, "Sur l'École de Francfort ", Cités, vol. 3, n 11, 2002, p. 147-155; Stephano Petrucciani, "La Théorie critique de l'école de Francfort et le mouvement des années 1968 : un rapport complexe ", Actuel Marx, $\mathrm{n}^{\circ}$ 48, $\mathrm{n}^{\circ}$ 2, 2010, p. 138-151; Olivier Voirol, «La Théorie critique de l'École de Francfort : une relecture ", Mouvements, n 61, 2010, , p. 23-32. 
France, d'une part, la Grande-Bretagne et les États-Unis, d'autre part. Laurent Béru met en évidence l'institutionnalisation de cette question, différemment déclinée dans les programmes universitaires ou dans les divers champs académiques. Il parle de "décalage [...de] déphasage académique ${ }^{4}$ » entre la France et les États-Unis, car ces derniers développent la recherche sur les Ethnic studies, les Subaltern studies, ou encore les Minority studies, certaines de ces études résultant d'ailleurs directement ou indirectement des conflits politiques et sociaux qui ont traversé le pays. Certains principes politiques (Affirmative Action, Civil Rights) ont déterminé par la suite des changements effectifs pour - par exemple - la représentation des Noirs à la télévision.

Il est donc difficile de comprendre ce désintérêt français sur la question des minorités ethniques, quand la Grande-Bretagne pays pourtant proche géographiquement et productif en matière de recherche en sciences sociales - et les États-Unis connaissent depuis plusieurs années déjà un changement de paradigme et de perspective sur cette question. La méfiance et la distance prises par la majeure partie de la communauté scientifique française pour ce qui remet en cause une certaine posture intellectuelle se voient clairement : "Il faudra attendre le passage du millénaire pour voir se développer dans le monde académique français journées et colloques sur les cultural studies et leurs déclinaisons et connexions ${ }^{5} »$. Les chercheurs qui ont travaillé sur l'accueil des cultural studies en France déclinent les raisons de la "réticence" de la communauté scientifique de diverses manières. Est d'abord relevé le principe de " la place est prise ${ }^{6}$ » en référence à la place historiquement et majoritairement occupée par l'histoire et par la sociologie pour les questions relatives aux études culturelles. Sur le même plan scientifique, Françoise Albertini et Nicolas

$\overline{4}$ Laurent Béru, «Différences d'approche entre traditions française et nordaméricaine dans les recherches en ethnic/race studies ", dans Françoise Albertini et Nicolas Pelissier (dir.), Les Sciences de l'information et de la communication à la rencontre des cultural studies, Paris, LHarmattan, 2009, p. 131.

5 Erik Neveu, op. cit., p. 315.

$6 \quad$ Erik Neveu, op. cit., p. 332. 
Pélissier précisent que la sémiologie d'inspiration structuraliste rechigne à mettre en relation le texte et le public, au contraire des cultural studies, et supposent que joue aussi « la prégnance d'un imaginaire républicain français hostile à une conception de la société en termes de minorités (ethniques, linguistiques, sexuelles, etc. $)^{7}$ ». Une autre raison tient à la barrière linguistique : la langue anglaise n'est que peu traduite, même si, comme le soulignent Erik Neveu et Armand Mattelart, les fondateurs des cultural studies ont à l'origine importé les théories françaises ${ }^{8}$. Nous posons d'ailleurs comme postulat que c'est pour ces différentes raisons que la France n'a que tardivement travaillé sur la notion de minorité ethnique à la télévision, expliquant de fait la jeunesse des travaux universitaires menés sur le sujet.

\section{En France}

Le concept de représentation des minorités ethniques est d'abord étudié par des chercheurs en sociologie dont les analyses mettent en évidence la " mal-représentation " et la "sur-stigmatisation" " des publics concernés, la médiatisation « simpliste et globalisante $^{10}$ » dont souffrent des communautés ciblées, ou encore leur sous-représentation ${ }^{11}$ - au détriment de leur intégration dans l'espace public. Sur le plan scientifique, le terme même de " minorité ethnique " connaît une déclinaison assez large en fonction des critères retenus pour qualifier les personnes concernées : blancs/non-blancs, critères en fonction de la couleur de peau et des caractéristiques physiques, noirs/maghrébins arabes/

\footnotetext{
$\overline{7}$ Françoise Albertini et Nicolas Pélissier, , op. cit., p. 18.

8 Armand Mattelart et Erik Neveu, op. cit.

9 Isabelle Rigoni, «Les Médias des minorités ethniques. Représenter l'identité collective sur la scène publique ", Revue européenne des migrations internationales, vol. 26, n 1, 2010, p. 8.

10 Claire Cossée, "Médias tsiganes en France et en Hongrie. Re-présentation de soi dans l'espace public ", Revue européenne des migrations internationales, vol. 26, $\mathrm{n}^{\circ} 1,2010$, p. 58.

11 Magali Nayrac, "La Question de la représentation des minorités dans les médias, ou le champ médiatique comme révélateur d'enjeux sociopolitiques contemporains ", Cahiers de l'Urmis, n 13, octobre 2011, http://urmis.revues. org/index1054.html, site consulté en septembre 2012.
} 
asiatiques, etc. ${ }^{12}$

La première publication scientifique traitant de la représentation des minorités dans les médias date de 1971, avec l'ouvrage de Françoise Birnholz et Giuseppe Callovi. Claire Frachon et Virginie Sassoon le rappellent dans leur ouvrage paru en 2009, dont l'un des objectifs vise à identifier les travaux scientifiques et les productions médiatiques qui ont traité de la représentation des minorités ethniques depuis les vingt dernières années. Les références bibliographiques relevées permettent de baliser assez clairement le cheminement suivi par les chercheurs français depuis 1980 et la fréquence des publications reliées à cette thématique. Les auteures relèvent que "les science sociales restent muettes $^{13}$ " sur le sujet. Les années 1990, quant à elles, marquent un changement en raison de la production scientifique un peu plus importante sur le sujet : la revue Migration Société du Centre d'information et d'études sur les migrations internationales (CIEMI) y consacre régulièrement des numéros, à l'image d'autres revues spécialisées (Migrants-Formation ou MediaScope). Dans le cadre de travaux universitaires, on ne relève pas de thèses sur le sujet, si ce n'est le travail de Marie-France Malonga qui consacre principalement son diplôme d'études approfondies (DEA), en 2000, ainsi que sa thèse, en 2007, à la question de la représentation des minorités ethniques.

Ces études restent cependant majoritairement à dominante sociologique, avant de s'ouvrir dans les années 2000 à d'autres disciplines comme les Sciences de l'information et de la communication. Guy Lochard, notamment, coordonne un dossier en

12 Marie-France Malonga, "Télévision française et intégration. Présence et représentation des minorités visibles à l'écran ", mémoire de DEA, Université Panthéon-Assas, Institut Français de Presse, 2000; Guy Lochard (dir.), Minorités visibles, Médiamorphoses, $\mathrm{n}^{\circ} 17$, septembre 2006; Observatoire de la diversité dans les médias audiovisuels (CSA), Représentation de la diversité dans les programmes de télévision, 2008, coordonné par Éric Macé; Catherine Ghosn, "Représentation de la diversité à la télévision : à partir de quelles normes?", Sciences de la Société, n 81, 2010, p. 27-43. 
$2006^{14}$ et choisit une approche comparative pour évaluer la représentation des minorités ethniques dans quatre pays francophones (la France, la Suisse, le Canada, la Belgique) et identifier les réglementations, les dispositifs mis en œuvre dans ces pays. En sciences de l'information et de la communication, cette recherche reste cependant isolée. Des groupes de recherche lui donneront par la suite plus de poids et de visibilité par leur visée internationale (le groupe Minority Media, le projet de recherche, Mediamigraterra). Ces recherches restent majoritairement limitées au domaine universitaire et il faut attendre mai 2000 pour consulter une étude menée par une chercheure (Marie-France Malonga) destinée au Conseil Supérieur de l'Audiovisuel (CSA) qui en est le commanditaire. Cette étude vise comme objectif à "dresser un état des lieux de la place réservée aux différentes composantes de la communauté nationale [et] faire une analyse détaillée de la programmation des chaînes hertziennes françaises afin d'évaluer la présence des minorités ethniques sur le petit écran ${ }^{15}$ ». Huit ans s'écoulent avant la publication d'un second document de synthèse coordonné par un autre chercheur, Éric Macé, et destiné à l'Observatoire de la diversité dans les médias audiovisuels (crée par le CSA). Cette synthèse vise le double objectif de "s'assurer que le traitement télévisuel des individus et des groupes n'est pas discriminant [et] veiller à ce que le traitement télévisuel des individus et des groupes sujets à discrimination ne reflète pas les préjugés et les stéréotypes ${ }^{16}$ ". À ce jour, ces deux synthèses sur la représentation des minorités ethniques à la télévision restent les seules menées par des scientifiques pour des instances audiovisuelles.

Quelles répercussions ces démarches et résultats scientifiques ont-ils concrètement sur la politique télévisuelle? Sur un plan général, nous nous appuyons sur deux postulats pour considérer que quelques disciplines de sciences sociales en France pourraient

\footnotetext{
14 Guy Lochard, "Minorités visibles », op. cit.

15 Marie-France Malonga, op. cit. p. 2.

16 Observatoire de la diversité dans les médias audiovisuels (CSA), Représentation de la diversité dans les programmes de télévision, 2008, coordonné par Éric Macé, p. 4.
} 
permettre de réorienter dans une certaine mesure la politique télévisuelle des chaînes quant à la représentation des minorités ethniques.

\section{Les postulats}

Le premier postulat se réfère à l'avis adopté par des chercheurs soulignant que la télévision a un impact dans la construction de l'identité car elle affirme une "visibilité " et une existence reconnue, avérée dans l'espace public ${ }^{17}$. Ce processus de stratégie identitaire se pose même, pour Marc Lits, comme un postulat de base :

Toute recherche portant sur les contributions des médias à la formation ou au développement des identités nationales repose sur un postulat implicite selon lequel ces médias jouent un rôle déterminant dans la constitution des représentations sociales collectivement partagées ${ }^{18}$.

Le second postulat se réfère au cahier des charges de France Télévisions. Celui-ci fait suite à l'expression d'une volonté de politique audiovisuelle discutée entre les principaux protagonistes, fixée ensuite par un décret. Pour notre étude, nous faisons concrètement référence au « décret $n^{\circ} 2009-796$ du 23 juin 2009 fixant le cahier des charges de la société nationale de programme France Télévisions ». Ce décret souligne en Préambule la volonté de France Télévisions d'être exemplaire en ce qui concerne la représentation des minorités ethniques et contient dans la rubrique "La lutte contre les discriminations et la représentation de la diversité à l'antenne » (article 37) les affirmations suivantes :

France Télévisions prend en compte, dans la représentation à l'antenne, la diversité des origines et des cultures de la communauté nationale. [...] Dans le cadre des recommandations du Conseil supérieur de l'audiovisuel, la société met en œuvre les actions permettant d'améliorer la représentation de la diversité de la société française ${ }^{19}$.

17 Marie-France Malonga, op. cit:; Marc Lits, « Les Télévisions belges au carrefour européen ", dans Marie-Françoise Lévy et Marie-Noëlle Sicard (dir.), Les Lucarnes de l'Europe, Télévisions, cultures, identités, 1945-2005, Paris, Publications de la Sorbonne, 2008, p. 139-149.

19 Article 37, décret $n^{\circ} 2009-796$ du 23 juin 2009 fixant le cahier des charges de la société nationale de programme France Télévisions, http://www.legi- 
Il est à noter la différence de termes utilisés dans les recherches universitaires, d'une part, dans les rapports institutionnels émanant des instances télévisuelles, d'autre part. $\mathrm{Si}$ " minorité ethnique " apparaît littéralement dans les études scientifiques citées, il se dote d'une signification plus large et certainement volontairement plus informelle dans les comptes-rendus de France Télévisions et du CSA, alors même qu'ils renvoient aux mêmes différences physiques qui s'écartent du modèle de référence (blanc, de type occidental). L'affirmation consistant à mieux représenter les minorités ethniques à la télévision est ici formalisée, institutionnalisée et succède aux différents travaux que nous avons relevés dans le champ des différentes productions scientifiques. Il reste à évaluer dans quelles mesures ces intentions, déjà affirmées dans les précédents cahiers des charges, modifient effectivement le paysage audiovisuel.

\section{Les rapports des instances audiovisuelles}

En ce qui concerne précisément la problématique de la représentation des minorités à la télévision, le CSA s'est vu confier par la loi de $2006^{20}$ la tâche de veiller à la représentation de la diversité de la société française dans les programmes audiovisuels. Cette loi :

attribue au CSA la mission, d'une part, de contribuer aux actions en faveur de la cohésion sociale et à la lutte contre les discriminations dans le domaine de la communication audiovisuelle et, d'autre part, de veiller, notamment auprès des éditeurs de communication audiovisuelle, compte tenu de la nature de leurs programmes, à ce que la programmation reflète la diversité de la société française ${ }^{21}$.

La loi du 5 mars 2009 relative à la communication audiovisuelle et au nouveau service public de la télévision consolide la responsabilité du CSA sur le sujet et exprime des attentes vis-à-vis

france.gouv.fr/affichTexte.do?cidTexte=JORFTEXT000020788471\&fastPos $=1 \&$ fastReqId $=1553707249 \&$ categorieLien=id\&oldAction=rechTexte, site consulté en septembre 2012.

20 Loi pour l'égalité des chances.

21 Cf. :http://www.csa.fr/Television/Le-suivi-des-programmes/La-representationde-la-diversite/Quel-est-le-role-du-Conseil-dans-la-promotion-de-ladiversite. 
des éditeurs, surtout vis-à-vis du service public (France Télévisions). De manière pratique, le CSA a mis en place deux types de projet. Le premier prévoit de rendre une synthèse annuelle au Parlement, rendant compte des initiatives prises par les différents opérateurs au sujet de la représentation des minorités. Le second concerne la réalisation d'un baromètre biannuel dont l'objectif vise à évaluer la perception de la "diversité » à la télévision, diversité, pris ici au sens large (femmes / hommes / minorités ethniques / personnes handicapées /...).

Le rapport du CSA remis au Parlement en avril 2010 constitue un compte-rendu des initiatives prises aussi bien par les éditeurs que par ce Conseil sur le sujet de la représentation des minorités ethniques à la télévision. Le rapport se décompose en quatre principales parties : une consacrée aux initiatives des éditeurs hors antenne et à l'antenne (bilan des chaînes, analyse d'une semaine de programme), les trois dernières se rattachant essentiellement aux actions entreprises par le CSA. Le deuxième rapport émane du Comité permanent de la diversité22, dont les missions ont trait à :

- la promotion de la représentation des minorités aussi bien à l'écran qu'au sein de l'entreprise,

- la mise en place d'indicateurs de suivi de la présence des minorités (au sein de l'entreprise et dans les programmes).

Publié en avril 2010, le rapport paraît à la même date que celui du CSA et recense, entre autres, la problématique des minorités à la télévision depuis les dix dernières années. La publication de ces rapports prend place dans un contexte bien précis : sur le plan de la politique audiovisuelle, elle naît de la volonté générale de mieux représenter les minorités ethniques à la télévision, projet formalisé et légalement reconnu. Sur le plan de la recherche scientifique et universitaire française, ces rapports succèdent à des publications qui présentent un travail sur l'aspect conceptuel de la visibilité ou de l'invisibilitée ${ }^{23}$ et de la représentation des

22 Le Comité permanent de la diversité a été mis en place par France Télévisions en juin 2009.

23 Olivier Voirol, op. cit.; Wayne Breckhus, "Une Sociologie de l'invisibilité : 
minorités ${ }^{24}$. Sur le plan social, quelques événements font aussi date. Le premier nous ramène à la fin des années 1990 où Calixthe Beyala, fondatrice du Collectif Égalité, dépose une plainte contre le CSA et contre le gouvernement français en raison de l'insuffisance de la présence de Noirs à la télévision. Les émeutes de 2005, relatives aux violences urbaines qui se sont déroulées dans plusieurs banlieues françaises mettent en avant la problématique de la contestation sociale et celle du lien entre les minorités et les médias. Suite à cela, le Président de la République française a rencontré les responsables des chaînes nationales pour proposer des améliorations sur le thème de la représentation des minorités à la télévision. Ces rencontres ont permis de mettre en place la modification des missions et des obligations du CSA avec, par exemple, la diffusion de France $\hat{\mathrm{O}}^{25}$ sur le territoire métropolitain. En 2006, le gouvernement intègre au projet pour l'égalité des chances entre les Français des dispositions législatives particulières dans le but d'améliorer la représentation des minorités ethniques dans les médias audiovisuels.

Le contexte dans lequel s'inscrivent les rapports du CSA et de France Télévisions est donc riche en facteurs déterminants, mais le doute reste encore sur le rôle qu'ont pu jouer les sciences sociales sur les éventuelles avancées. Pour ces rapports, notre analyse porte sur quatre principales entrées : l'origine de l'enquête, l'objectif visé, la méthodologie adoptée et les particularités de ces enquêtes.

En ce qui concerne l'origine de l'enquête, le rapport du CSA ne mentionne nulle part les raisons qui en motivent la conduite, mais s'appuie sur quelques allusions. Il est simplement mentionné dans les premières pages que la préoccupation du CSA concernant un meilleur reflet de la représentation des minorités à la

réorienter notre regard ", Réseaux, nos 129-130, 2005, p. 243-272.

Guy Lochard, op. cit.; Marie-France Malonga, op. cit., Éric Macé, « Mesurer les effets de l'ethnoracialisation dans les programmes de télévision : limites et apports de l'approche quantitative de la diversité ", Réseaux, $\mathrm{n}^{\text {os }}$ 157-158, 2009, p. 237-265.

25 Sur le site de France Télévisions, France Ô est présentée comme la « chaîne des cultures métissées " qui " vise à faire découvrir et à faire partager au plus grand nombre la richesse et l'actualité de nos outremers ». 
télévision émane d'une "demande sociale croissante dans ce domaine » sans pour autant citer les références qui s'y rapportent ou bien même plus de précisions. Le rapport du Comité permanent de la diversité se révèle, quant à lui, plus explicite sur les origines de sa rédaction car il dresse un historique en guise d'explications reliées à l'origine de cette publication et rappelle à force de chiffres et de projets élaborés depuis dix ans les actions mises en place par France Télévisions ou encore par le CSA pour améliorer la représentation des minorités à la télévision. Sont ainsi énoncés les émeutes de 2005, le Plan d'Action Positive pour l'Intégration ${ }^{26}$, la création du Comité permanent de la diversité... Sous couvert de rappel de toutes ces informations, le rapport procède finalement de la même discrétion pour tout ce qui remonte aux origines sociales, politiques ou scientifiques de la problématique "représentation des minorités ethniques à la télévision ".

Dans un second temps, le rapport du CSA ne mentionne aucun objectif et se caractérise plutôt comme une synthèse de toutes les actions entreprises par le Conseil depuis l'année 2000, s'apparentant ainsi davantage à un compte-rendu technique. La seule séquence introductive ayant trait au contenu du rapport se résumé à quelques lignes précédant la première partie et annonce le plan : "La première partie du présent rapport présente [...]. La deuxième partie concerne [...]. La troisième partie traite $[\ldots]^{27} »$. Le rapport publié par le Comité permanent de la diversité procède par la même étape au début, car il se rapporte à un état des lieux effectué dans les structures, à l'antenne et dans les contenus, mais définit ensuite les objectifs déterminés à partir de ce constat. Les membres du Comité émettent ainsi des propositions qui se rapportent à trois volets : les structures de l'entreprise, la visibilité à l'antenne et le contenu des programmes, chacun de ces volets étant complété par plusieurs préconisations.

26 Ce plan a été lancé par France Télévisions en janvier 2004, avec l’objectif suivant : faire en sorte que la diversité de la société française soit visible sur toutes les chaînes de service public, mais aussi derrière les écrans.

27 CSA, Représentation de la diversité de la société française à la télévision. Rapport au Parlement, avril 2010, p.9. 
L'objectif visé par cette étude s'exprime au cours des toutes dernières lignes de l'introduction : "Dans un esprit d'efficacité et de clarté, les propositions du Comité s'articulent autour de trois axes [...]. Pour plus de lisibilité, chacun de ces axes fera l'objet de quatre préconisations, formant ainsi un ensemble d'une douzaine de mesures ${ }^{28} \%$.

La méthodologie sur laquelle reposent aussi bien les préconisations que les résultats de l'enquête se répartit en deux groupes. Le premier se caractérise par une approche synthétique et chronologique des actions entreprises sur le plan politique par les responsables des instances télévisuelles (CSA), avec une référence presque systématique à des textes de loi (CSA, Comité permanent de la diversité). Le second présente les projets mis en place avec d'autres institutions (CSA, Comité permanent de la diversité). Le rapport du CSA cite fréquemment les différents articles ou les lois qui lui donnent autorité pour travailler sur la représentation des minorités ethniques à la télévision : "L'article 3-1 de la loi du 30 septembre 1986 [...] confie au Conseil Supérieur de l'audiovisuel ${ }^{29}$... »; « la loi du 5 mars 2009 [...] a renforcé le rôle du Conseil Supérieur de l'audiovisue ${ }^{30} \ldots$... Pour ces deux instances, la méthodologie procède par une synthèse relevant les décisions d'ordre politique ou stratégique du groupe concerné, avec des échanges conventionnels mis en place entre elles ou encore par des auditions de professionnels de la télévision.

Les deux rapports adoptent un choix méthodologique assez étonnant pour ce qui concerne la contribution des publications qui ont précédé leurs bilans. On ne trouve nulle trace des enquêtes, études, rapports, etc. effectués avant eux. Ils citent essentiellement les publications dont le Conseil Supérieur de l'Audiovisuel se trouve à l'origine ou encore les initiatives qu'il a prises. La lecture de ces documents nous permet d'affirmer que, sans compter les références portant leurs signatures, le CSA et France Télévisions ne mentionnent pas les publications existantes

\footnotetext{
$28 \quad$ Comité permanent de la diversité, Rapport 2010, p. 4.

29 Comité permanent de la diversité, op. cit., , p. 7.

30 Ibid., p.7.
} 
sur le sujet. Ce manque de recul et le choix de ne limiter les références qu'à celles de leur périmètre ne peuvent qu'offrir une étude partielle de la représentation des minorités ethniques à la télévision, ignorant de manière volontaire ou pas les contributions antérieures qui pourraient apporter de précieux éléments. France Télévisions ne signale aucun autre rapport, si ce n'est celui du CSA. Sur le plan méthodologique, force est de constater l'absence de considération des diverses contributions portant sur le sujet, ou en tout cas l'insuffisance de prise en compte.

L'objet de notre travail consistait à étudier la production scientifique traitant de la représentation des minorités ethniques à la télévision en France, pour évaluer ensuite la manière dont le CSA et France Télévisions l'avaient intégrée dans la mise en place de leurs projets. On tire comme principale conclusion de cette étude que, sur le plan de la recherche scientifique en France, plusieurs chercheurs travaillent sur le concept des minorités ethniques, en mettant ces thématiques en lien avec la télévision. Principalement traitée en sociologie, puis, plus tardivement, en Sciences de l'information et de la communication - mais pas exclusivement -, cette problématique semble limiter sa production au seul champ de la recherche en sciences sociales, sans parvenir à partager les résultats avec les instances politiques et audiovisuelles. Le CSA et France Télévisions ont cependant initié des travaux d'envergure dont l'objectif était ou est encore d'œuvrer à une meilleure représentation des minorités à la télévision, comme le Plan d'Action Positive Pour l'Intégration en 2004, la mise en place du groupe de travail « Diversité » en 2007 au CSA, la création de l'observatoire de la diversité en 2008, le baromètre de la diversité en 2009, etc.; mais force est de constater que, même si des chercheurs ont travaillé pour le CSA, on ne peut que relever la lenteur ou la rareté des effets produits. 


\section{Bibliographie}

Albertini, Françoise et Nicolas Pélissier, Les Sciences de l'information et de la communication à la rencontre des cultural studies, Paris, L'Harmattan, 2009.

Béru, Laurent, « Différences d'approche entre traditions française et nordaméricaine dans les recherches en ethnic/race studies ", dans Françoise Albertini et Nicolas Pelissier (dir.), Les Sciences de l'information et de la communication à la rencontre des cultural studies, Paris, L'Harmattan, 2009, p. 131.

Birnholz Françoise et Giuseppe Callovi, Mass-média et immigration en France, École Française d'Attachés de Presse, Paris, 1971.

Breckhus, Wayne, "Une Sociologie de l'invisibilité : réorienter notre regard ", Réseaux, n 129-130, 2005, p. 243-272.

Cossée, Claire, « Médias tsiganes en France et en Hongrie. Re-présentation de soi dans l'espace public ", Revue européenne des migrations internationales, vol. 26, $\mathrm{n}^{\circ} 1,2010$, p. 57-80.

Frachon, Claire et Virginie Sassoon, Médias et diversité. De la visibilité aux contenus, Paris, Institut Panos Paris / Karthala, 2009.

Ghosn, Catherine, «Représentation de la diversité à la télévision : à partir de quelles normes? ", Sciences de la Société, nº 81, 2010, p. 27-43.

Glevarec, Hervé, Éric Macé et Éric Maigret, Cultural studies : anthologie, Paris, Armand Colin, 2008.

Lits, Marc, "Les Télévisions belges au carrefour européen ", dans MarieFrançoise Lévy et Marie-Noëlle Sicard (dir.), Les Lucarnes de l'Europe, Télévisions, cultures, identités, 1945-2005, Paris, Publications de la Sorbonne, 2008, p. 139-149.

Lochard, Guy (dir.), Minorités visibles, Médiamorphoses, ${ }^{\circ} 17$, septembre 2006.

Macé, Éric, « Mesurer les effets de l'ethnoracialisation dans les programmes de télévision : limites et apports de l'approche quantitative de la diversité ", Réseaux, nos 157-158/2009, p. 237-265.

Malonga, Marie-France, "Télévision française et intégration. Présence et représentation des minorités visibles à l'écran ", mémoire de DEA, Université Panthéon-Assas, Institut Français de Presse, 2000.

Mattelart, Armand et Erik Neveu, Introduction aux cultural studies, Paris, La Découverte, 2003.

Nayrac, Magali, «La Question de la représentation des minorités dans les médias, ou le champ médiatique comme révélateur d'enjeux sociopolitiques contemporains ", Cahiers de l'Urmis, n 13, octobre 2011, 
http://urmis.revues.org/index1054.html, site consulté en septembre 2012.

Neveu, Erik, «Les Voyages des cultural studies ", L'Homme, vol. 187-188, $\mathrm{n}^{\text {os }} 3-4,2008$, p. 315-341.

Petrucciani Stephano, «La Théorie critique de l'École de Francfort et le mouvement des années 1968 : un rapport complexe ", Actuel Marx, vol. 48, n 2, 2010, p. 138-151.

Rigoni, Isabelle, "Les Médias des minorités ethniques. Représenter l'identité collective sur la scène publique ", Revue européenne des migrations internationales, vol. 26, $\mathrm{n}^{\circ}$ 1, 2010, p. 7-16.

Simonis, Martine, «Baliser les pratiques professionnelles », Médiamorphoses, $\mathrm{n}^{\circ} 17$, septembre 2006, p.30-33.

Voirol, Olivier, "Visibilité et invisibilité : une introduction ", Réseaux, $\mathrm{n}^{\text {os }} 129-130,2005$, p. 9-36.

Voirol, Olivier, "La Théorie critique de l'École de Francfort : une relecture ", Mouvements, n $^{\circ}$ 61, 2010, p. 23-32.

Walter, Benjamin, "Sur l'École de Francfort ", Cités, vol. 3, n 11, 2002, p. $147-155$.

\section{Rapports, études}

CIEMI, Présence et représentation des immigrés et des minorités ethniques à la télévision française, 1991.

Club Averroes, Rapport 2009. La diversité dans les médias.

Comité permanent de la diversité, Rapport 2010.

CSA, Présence et représentation des minorités visibles à la télévision française, 2000, coordonné par M.F. Malonga.

CSA, Représentation de la diversité de la société française à la télévision. Rapport au Parlement, avril 2010.

Observatoire de la diversité dans les médias audiovisuels (CSA), Représentation de la diversité dans les programmes de télévision, 2008, coordonné par Éric Macé. 